\title{
Programa de intervención dietético-nutricional para la promoción de la salud en el lugar de trabajo en una empresa de la ciudad de Huesca, España
}

\author{
Marta Munar-Gelabert ${ }^{a, *}$,José Puzo-Foncillas ${ }^{b}$, Teresa Sanclemente $^{a}$

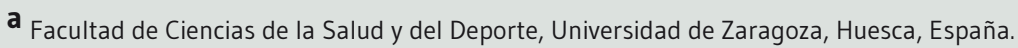 \\ b Facultad de Ciencias de la Salud y del Deporte, Universidad de Zaragoza, Huesca, España. Servicio de Bioquímica Clínica, \\ Hospital Universitario Miguel Servet, Zaragoza, España. \\ *martamunargelabert@gmail.com
}

Recibido el 6 de julio de 2015; aceptado el 12 de noviembre de 2015.

\begin{abstract}
PALABRAS CLAVE
Hábitos alimentarios;

Dieta;

Lugar de trabajo;

Promoción de la salud.
\end{abstract} Programa de intervención dietético-nutricional para la promoción de la salud en el lugar de trabajo
en una empresa de la ciudad de Huesca, España

\section{RESUMEN}

Introducción: La realización de programas de promoción de la salud en el lugar de trabajo puede mejorar el estado de salud de los trabajadores, disminuir el absentismo por enfermedad e incluso aumentar la productividad. El objetivo de este trabajo fue llevar a cabo una intervención dietético-nutricional en una empresa con 35 trabajadores de Huesca, con comedor propio, y valorar la eficacia de la misma.

Material y Métodos: Tras la valoración de los hábitos alimentarios de los trabajadores mediante un cuestionario autocontestado, se llevó a cabo un asesoramiento en la planificación de los menús del comedor y un plan de formación compuesto por tres talleres. Al finalizar, se compararon los resultados pre y post intervención de las variables estudiadas.

Resultados: Al inicio, la mitad de los encuestados realizaba cinco comidas diarias pero el desayuno era mayoritariamente incompleto y el grupo presentaba bajo consumo de frutas y hortalizas y alto de carne y alimentos ocasionales. A pesar de la mediana participación en los talleres y que no se cumplieron completamente los menús propuestos, tras la intervención de tres meses, se observó una aparente mejora de los hábitos de consumo (ligeros incrementos en frutas, hortalizas, pescado y lácteos y descensos en carne). Además, los trabajadores consideraron los planes de formación satisfactorios.

Conclusiones: La intervención dietético-nutricional en esta empresa es compleja debido a su propia idiosincrasia y al corto periodo disponible. Aun así, los empleados han adquirido una mayor conciencia de la relación dieta y salud, además de ser una experiencia positiva para la empresa. 


\section{KEYWORDS}

Food habits;

Diet;

Workplace;

Health promotion.
Dietary-nutritional intervention program for health promotion in the workplace in a company of the city of Huesca, Spain

\section{ABSTRACT}

Introduction: The development of health promotion programs in the workplace can improve employers' health, decrease the absenteeism due to illness and even increase the productivity. The aim of this work was to develop a dietary-nutritional intervention and to assess its effectiveness in a company with 35 employers from Huesca, which has its own canteen.

Material and Methods: Canteen menus planning advice and a training program consisting in three workshops were conducted after staff dietary habits assessment through a self-filledin questionnaire. The pre and post intervention results of the different studied variables were compared at the end.

Results: At the beginning, half of the respondents ate five meals a day, but the breakfast was mostly incomplete. The sample was characterized by a low intake of fruit and vegetables and a high intake of meat and "occasionally" products. It was observed an improvement in food habits (slight increases in fruit, vegetables, fish and dairy products and decreases in meat) despite lower involvement in workshops and considering that not all the menus suggested were fulfilled after three months intervention. In addition, the workshops had been considered satisfactory by the staff.

Conclusions: Carrying out a dietary and nutritional intervention is complex in this company due to its own idiosyncrasy and the short period available. However, the staff has acquired more awareness about the relationship between diet and health and it is a positive experience for the company.

\section{CITA}

Munar-Gelabert M, Puzo-Foncillas J, Sanclemente T. Programa de intervención dietético-nutricional para la promoción de la salud en el lugar de trabajo en una empresa de la ciudad de Huesca, España. Rev Esp Nutr Hum Diet. 2015; 19(4): 189 - 196 de la maquetación. DOI: 10.14306/renhyd.19.4.168

\section{INTRODUCCIÓN}

Es bien conocido que existe una relación estrecha entre el estilo de vida, incluida la dieta, y diversas enfermedades crónicas ${ }^{1,2}$. Por ello, resulta imprescindible realizar programas de promoción de la salud (PPSs) en los diferentes grupos de población y valorar si éstos podrían llegar a reducir algunos de los problemas sanitarios relacionados ${ }^{3}$. Debido a que el $60 \%$ de las horas que se está despierto se pasan en el lugar de trabajo, la OMS ha afirmado que éste es uno de los ambientes más idóneos para llevar a cabo PPSs4.

Son numerosos los PPSs llevados a cabo en los lugares de trabajo con resultados prometedores tanto en la mejora de la salud como en la disminución del absentismo y el aumento de la productividad ${ }^{5-7}$. Sin embargo, existe una falta de información acerca de si estos resultados son aplicables a los países del sur de Europa ${ }^{6}$ y a empresas de pequeño tamaño ${ }^{8}$.

Este trabajo nace de la solicitud de la dirección de una empresa de 35 empleados situada en la ciudad de Huesca (España) de recibir asesoramiento especializado a nivel dietético-nutricional para la revisión de los menús ofertados en su comedor de reciente implantación, además de estar abierta a otras iniciativas que tuvieran como objetivo final la mejora de la salud de sus empleados.

De acuerdo con lo expuesto anteriormente, se procedió a llevar a cabo un programa de intervención dietético-nutricional en dicha empresa con el objetivo de que los trabajadores cumplieran las recomendaciones de alimentación saludable y valorar la eficacia del mismo. 


\section{MATERIAL Y MÉTODOS}

La metodología utilizada para llevar a cabo este trabajo se esquematiza en la Figura 1 y se describe en los apartados siguientes. Se trata de una intervención dietético-nutricional que, siguiendo las indicaciones de la dirección, incluyó a todos los trabajadores de la empresa.

\section{Descripción de la población estudiada y detección de las necesidades}

Se utilizó un cuestionario con el objetivo de valorar el estado nutricional y los hábitos alimentarios de los trabajadores y, posteriormente, se analizaron los datos obtenidos con el fin de obtener una descripción de la población sobre la que se iba a trabajar que permitiera detectar las necesidades existentes $y$, por tanto, diseñar un PPS ad hoc.
Atendiendo a las indicaciones de la empresa, el cuestionario fue autocontestado, en grupos de cinco personas, en presencia de la dietista-nutricionista responsable del PPS que resolvió las dudas de los participantes. Dicho cuestionario fue totalmente anónimo y confidencial por lo que se asignó un código de identificación a cada empleado que se mantuvo en el resto de cuestionarios y/o documentos que se les administró. Contestar al cuestionario, tras la previa explicación del programa de intervención, suponía otorgar el correspondiente consentimiento informado por parte del participante.

La primera parte del cuestionario se elaboró específicamente para este estudio con preguntas acerca de: edad, sexo, peso y talla autorreferidos a partir de los cuales se calculó el Índice de Masa Corporal (IMC) (como peso [kg] entre talla al cuadrado [m]), horario laboral, historia clínica (personal y familiar) y hábitos dietéticos cualitativos (fraccionamiento de las ingestas, alimentos ingeridos en las colaciones de

Figura 1. Cronograma explicativo de las actividades relativas a la asesoría en la planificación de menús y a la realización de los talleres de los planes de formación llevados a cabo durante el programa de intervención dietético-nutricional así como a las valoraciones inicial y final.

\begin{tabular}{|c|c|c|c|c|}
\hline NOVIEMBRE & DICIEMBRE & ENERO & MARZO & ABRIL / MAYO \\
\hline $\begin{array}{l}\text { VALORACIÓN } \\
\text { SITUACIÓN } \\
\text { INICIAL: } \\
\text { Los menús ofertados en } \\
\text { el comedor no se hacen } \\
\text { en base a una planilla. } \\
\text { Recopilación de las } \\
\text { fichas de plato de las } \\
\text { recetas del comedor. } \\
\text { Compilación de un } \\
\text { cuestionario por parte } \\
\text { de los empleados. } \\
\text { Análisis de los resulta- } \\
\text { dos de los cuestionarios } \\
\text { y, consecuentemente, } \\
\text { establecimiento de los } \\
\text { objetivos de los planes } \\
\text { de formación. }\end{array}$ & $\begin{array}{l}\text { ASESORÍA } \\
\text { COMEDOR: } \\
\text { Elaboración de cuatro } \\
\text { planillas. Cada } \\
\text { semana existen dos } \\
\text { opciones de menús } \\
\text { diarias (tal y como } \\
\text { tienen costumbre } \\
\text { en el comedor de } \\
\text { la empresa). Dichas } \\
\text { planillas se prevé que } \\
\text { puedan ser utilizadas } \\
\text { posteriormente. } \\
\text { Calibración (EasyDiet@) } \\
\text { y revisión de las } \\
\text { recetas del comedor a } \\
\text { partir de las fichas de } \\
\text { plato aportadas por el } \\
\text { cocinero. } \\
\text { Ejemplos de menús } \\
\text { con recetas nuevas, } \\
\text { los cuales se comentan } \\
\text { con el cocinero de la } \\
\text { empresa para que } \\
\text { opine respecto a su } \\
\text { aplicabilidad. }\end{array}$ & $\begin{array}{l}\text { MENÚS DEL } \\
\text { COMEDOR } \\
\text { TALLER 1: ¿Qué } \\
\text { comemos?, ¿Cómo } \\
\text { deberíamos hacerlo? } \\
\text { Temas tratados: } \\
\text { Concepto de dieta } \\
\text { saludable y ración. } \\
\text { Pirámide de los } \\
\text { alimentos de la } \\
\text { SENC. } \\
\text { Distribución } \\
\text { temporal de } \\
\text { las comidas y } \\
\text { composición de las } \\
\text { mismas (Desayuno } \\
\text { y almuerzo). } \\
\text { Material entrega- } \\
\text { do: Tríptico a cada } \\
\text { empleado, el cual } \\
\text { resume el contenido } \\
\text { del plan de formación. }\end{array}$ & $\begin{array}{l}\text { TALLER 2: ¿Sabemos } \\
\text { qué comemos? } \\
\text { Temas tratados: } \\
\text { Recordatorio } \\
\text { composición de las } \\
\text { diferentes comidas. } \\
\text { Legumbres, carne, } \\
\text { pescado y fruta: } \\
\text { información básica } \\
\text { sobre su composi- } \\
\text { ción, características } \\
\text { propias y distribución } \\
\text { idónea. } \\
\text { Material entregado: } \\
\text { Póster DIN-A3 que } \\
\text { contiene la información } \\
\text { relacionada con el plan } \\
\text { de formación para que } \\
\text { la la empresa lo cuelgue } \\
\text { en un lugar visible. }\end{array}$ & $\begin{array}{l}\text { TALLER 3: jElijamos } \\
\text { el menú! } \\
\text { Recordatorio de los dos } \\
\text { talleres más consejos } \\
\text { necesarios para elegir } \\
\text { correctamente el } \\
\text { menú disponible en la } \\
\text { empresa. } \\
\text { Temas tratados: } \\
\text { Importancia del } \\
\text { desayuno /almuerzo } \\
\text { y su composición. } \\
\text { Opciones de segundo } \\
\text { plato: carne, pescado } \\
\text { y legumbres. } \\
\text { Importancia de la } \\
\text { fruta y verdura. } \\
\text { Concepto de } 5 \text { al día. } \\
\text { Concepto de ración. } \\
\text { Actividad práctica: } \\
\text { elección de las opciones } \\
\text { saludables de menús } \\
\text { en base a la oferta del } \\
\text { comedor de la empresa. } \\
\text { VALORACIÓN DE } \\
\text { LA EFICACIA DEL } \\
\text { PROGRAMA }\end{array}$ \\
\hline
\end{tabular}


Programa de intervención dietético-nutricional para la promoción de la salud en el lugar de trabajo en una empresa de la ciudad de Huesca, España

la mañana, picoteo entre horas). La segunda parte incluyó cuestiones acerca de la frecuencia de consumo de "alimento/grupo de alimentos" basadas en el cuestionario del Programa PERSEO de la Estrategia NAOS (Ministerio de Sanidad y (Consumo) ${ }^{9}$, adaptadas para adultos. Las respuestas obtenidas en esta segunda parte se recodificaron tomando como criterio el cumplimiento de la recomendación de consumo de los diferentes grupos de alimentos para la población española de la Sociedad Española de Nutrición Comunitaria (SENC) ${ }^{10}$, de modo que se clasificaron como: "cumple", "no cumple por menor consumo" y "no cumple por mayor consumo". Para las variables cuantitativas se calcularon la media y la desviación típica; y para las categóricas, las correspondientes tablas de frecuencias.

El diseño del estudio así como su ejecución se realizaron de acuerdo a los principios éticos descritos en la Declaración de Helsinki.

\section{Planificación de la intervención dietético-nutricional}

Planificación de menús y asesoría a cocina: La planificación de los menús se efectuó a partir de cuatro planillas diseñadas por la dietista-nutricionista y de las fichas de plato de cada una de las recetas que se realizaban en el comedor de la empresa, convenientemente calibradas para conocer su composición nutricional. Las planillas confeccionadas se comentaron con el cocinero de la empresa para que opinara sobre su aplicabilidad en el comedor. El resultado final fue el diseño de los menús semanales con dos opciones de primer y segundo plato para cada uno de los cinco días laborables de la semana. Se propuso aplicarlas en el comedor a partir de enero.

Plan de formación: A partir de los resultados obtenidos con el cuestionario inicial y la información relacionada con el funcionamiento de la empresa, se planificaron tres talleres (entre la $1^{\text {a }}$ semana de enero y la $1^{\text {ạ }}$ semana de abril) basados en una charla participativa llevada a cabo por la dietista-nutricionista más la entrega de material de apoyo (para los empleados y para la empresa) que se comentaba con los participantes. Los días elegidos para realizar cada sesión, de unos 30 minutos de duración, se acordaron en cada ocasión con la dirección de la empresa en función de la carga de trabajo prevista.

Respecto a los contenidos, se consideraron tres líneas: TaIler 1, aspectos básicos sobre una alimentación saludable según las actuales guías alimentarias para la población española ${ }^{10}$; Taller 2, los resultados de valoración del estado nutricional y hábitos alimenticios de los empleados según el cuestionario inicial; y Taller 3, algunos consejos necesarios para una buena elección de los menús disponibles en el comedor de empresa.

\section{Valoración de la eficacia}

Al mes de la realización del Taller 3, los empleados compilaron un segundo cuestionario, también elaborado para este fin, el cual valoraba aquellos aspectos que se habían incluido como objetivos de los talleres así como los cambios realizados en los menús. Además, se solicitó que indicaran en qué talleres habían participado. Finalmente, se incluyeron una serie de preguntas subjetivas para conocer si, desde el punto de vista de los empleados, el programa de intervención dietético-nutricional había resultado útil.

La eficacia del programa se estimó mediante la comparación de los resultados de los hábitos alimentarios descritos en este segundo cuestionario con los resultados iniciales, expresada como diferencia entre ambos datos, así como mediante la valoración de las respuestas a la serie de preguntas subjetivas.

\section{Resultados}

\section{Descripción de la población estudiada y objetivos del plan de formación}

De los 35 empleados de la empresa, contestaron al cuestionario inicial un total de 28 adultos de 32,5 $\pm 11,7$ años (rango de edad, 18-57 años) mayoritariamente hombres (93\%) con un IMC de $26,3 \pm 3,5 \mathrm{~kg} / \mathrm{m}^{2}$. La mayoría (75\%) manifestó estar preocupada por la comida y consideraba que la alimentación es importante en la salud (89\%). Es destacable que el 39\% presentaba alguna patología relacionada con la alimentación (hipertensión arterial, dislipemias, enfermedad cardiovascular, diabetes, enfermedades digestivas) y el $68 \%$ informó de antecedentes familiares de enfermedades relacionadas con la alimentación. Asimismo, el 71\% afirmó haber recibido consejos relacionados con la alimentación y en el $32 \%$ de los casos estaban relacionados con la patología que presentaban.

En relación con los hábitos alimentarios, la mitad de los sujetos realizaba las cinco comidas principales mientras que el resto se repartía de forma equitativa entre tres y cuatro comidas, siendo las colaciones almuerzo y merienda las que presentan una mayor variación en cuanto a su cumplimiento. Relacionado con esto, un $57 \%$ indicó que solía "picar entre horas".

Puesto que, de forma general, los empleados tenían un horario de trabajo desde las 7:30 hasta las 17:00 horas, se creyó necesario investigar el cumplimiento con las recomendaciones para las comidas realizadas por la mañana ${ }^{10}$. 
Programa de intervención dietético-nutricional para la promoción de la salud en el lugar de trabajo en una empresa de la ciudad de Huesca, España

Todos los individuos realizaban una ingesta por la mañana pero únicamente un $18 \%$ incluía los tres componentes básicos (lácteo, fruta y cereal) mientras que el 53\% remitió comer sólo dos (el 60\%, la combinación lácteo+cereal frente al 40\%, la combinación lácteo+fruta) y el resto únicamente tomaba lácteo (75\%) o fruta (25\%).

Respecto a la adecuación de consumo de los diferentes grupos de alimentos según las recomendaciones para la población española ${ }^{10}$, más del $50 \%$ de los encuestados presentaba una frecuencia de consumo menor de la recomendada para frutas y hortalizas y mayor para bollería y aperitivos. Aunque el número de individuos que cumplían las recomendaciones de la SENC para carne, lácteos, pescado, legumbres y pasta/arroz fue superior, se observó un elevado porcentaje de incumplimiento por alto consumo de carne.

Como consecuencia de estos resultados, los objetivos concretos del plan de formación basados en las líneas descritas en la metodología fueron (Figura 1): describir la Pirámide de Ios alimentos de la SENC; adecuar la distribución temporal de las comidas así como los componentes de desayuno, almuerzo y merienda; aumentar el consumo de frutas y hortalizas; profundizar en los grupos de alimentos utilizados como segundo plato; conocer los conceptos de "ración" y de "consumo ocasional"; y consejos necesarios para elegir correctamente el menú disponible en la empresa.

\section{Valoración de la eficacia del programa de intervención dietético-nutricional}

El cuestionario utilizado para obtener la información necesaria para la valoración de la eficacia del programa fue cum-

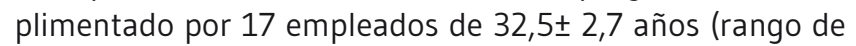
edad, 22-59 años) mayoritariamente hombres (88\%) con un IMC de $25,4 \pm 4,6 \mathrm{~kg} / \mathrm{m}^{2}$.

En relación al número de comidas realizadas de forma diaria, mientras que casi la mayoría (47\%) cumplió con la recomendación de realizar las cinco comidas, seguía existiendo un porcentaje elevado de sujetos que realizaban cuatro comidas diarias (29\%) o sólo tres (24\%). No hubo mejoras en este aspecto respecto a la situación inicial (Tabla 1).

Respecto a los componentes incluidos en las comidas de la mañana (Figura 2), tras la intervención, únicamente el 12\% de los empleados cumplió la recomendación de tomar tres componentes en el desayuno, ya que la inmensa mayoría se decantó por uno o dos componentes ( $41 \%$ en ambos casos). En el almuerzo estaba recomendada la ingesta de dos componentes, ello lo realizó un 35\% de los empleados, mientras que la mayor parte (47\%) únicamente incluyó uno (el 18\% no almuerza). Por consiguiente, no existió una notable mejoría.
Para la frecuencia de consumo de los diferentes alimentos, tal y como se muestra en la Tabla 1 , se realizó una valoración meramente cualitativa debido al pequeño tamaño de la muestra de individuos para los que se disponía de datos antes y después de la intervención. Respecto a estos resultados, cabe destacar la aparente mejoría en el cumplimiento de las recomendaciones de fruta y hortalizas, pescado, carne y lácteos aunque un porcentaje alto de individuos siguió teniendo consumos bajos de frutas, hortalizas cocinadas y lácteos. Respecto a las legumbres, el número de trabajadores con un consumo correcto disminuyó en casi un $30 \%$ aumentando la prevalencia de individuos con consumos menores a los recomendados.

Finalmente, si bien es cierto que más de la mitad de los empleados (64\%) no asistió a los tres planes de formación, el $53 \%$ de los empleados que contestaron al segundo cuestionario fue capaz de escribir tres consejos relacionados con la información sobre alimentación recibida entre los meses de enero y mayo (12\% únicamente recordaba uno, $24 \%$ no contestó). Además, todos los empleados remitieron que la información recibida resultaba de gran utilidad y el $71 \%$ de los mismos lo puso en práctica en contraposición al $29 \%$ que no lo hizo. Para comprobar que realmente así era, se preguntó sobre cuál era el aspecto que se había cambiado; el $47 \%$ lo mencionó, mientras que el $29 \%$ no fue capaz (el $24 \%$ no contestó a la cuestión). Es muy importante resaltar que todos los cambios tenían relación con aspectos que se habían comentado en los planes de formación, además de tener en cuenta que el $75 \%$ de ellos hizo alusión a los objetivos propuestos en dichos planes.

\section{DISCUSIÓN Y CONCLUSIONES}

La intervención dietético-nutricional se ha llevado a cabo con un éxito parcial en una empresa de 35 empleados, mayoritariamente hombres, donde la mayoría afirma ser consciente de la relación entre la dieta y el estado de salud. En general, remiten unos hábitos alimenticios parcialmente adecuados y la media del IMC indica un ligero sobrepeso. No se han conseguido mejoras importantes en el número de comidas que se realizan al día y en la diversidad de componentes de los desayunos y almuerzos, pero sí se ha detectado una pequeña mejora en la frecuencia de consumo de determinados grupos de alimentos así como en los conocimientos acerca de una alimentación saludable por parte de los trabajadores de esta empresa. Aunque los datos utilizados son autorreferidos, por lo que se podría pensar que los resultados estén sesgados, el hecho de que la descripción de la dieta que han proporcionado los 
Tabla 1. Comparativa de los resultados de las variables valoradas antes y después del programa de intervención dietético-nutricional. Datos expresados como número y porcentaje entre paréntesis (x (\%)). Las variaciones con signo + indican un aumento de la frecuencia y con signo -, una disminución.

\begin{tabular}{|c|c|c|c|c|c|c|c|c|c|c|c|c|}
\hline \multirow{2}{*}{$\begin{array}{l}\text { Número de } \\
\text { comidas día }\end{array}$} & \multicolumn{4}{|c|}{ VALORACIÓN INICIAL } & \multicolumn{4}{|c|}{ VALORACIÓN FINAL } & \multicolumn{4}{|c|}{ VARIACIÓN } \\
\hline & 5 & 4 & 3 & Picoteo & 5 & 4 & 3 & Picoteo & 5 & 4 & 3 & Picoteo \\
\hline & $10(59)$ & $4(23)$ & $3(18)$ & $7(41)$ & $8(47)$ & $5(29)$ & $4(24)$ & $8(47)$ & $-2(-12)$ & $+1(+6)$ & $+1(+6)$ & $+1(+6)$ \\
\hline $\begin{array}{l}\text { Frecuencia de } \\
\text { consumo de } \\
\text { alimentos }\end{array}$ & $\begin{array}{c}\text { Sí } \\
\text { cumplen }\end{array}$ & $\begin{array}{c}\text { No } \\
\text { cumplen } \\
\text { por } \\
\text { MENOR } \\
\text { consumo }\end{array}$ & $\begin{array}{c}\text { No } \\
\text { cumplen } \\
\text { por } \\
\text { MAYOR } \\
\text { consumo }\end{array}$ & & $\begin{array}{c}\text { Sí } \\
\text { cumplen }\end{array}$ & $\begin{array}{c}\text { No } \\
\text { cumplen } \\
\text { por } \\
\text { MENOR } \\
\text { consumo }\end{array}$ & $\begin{array}{c}\text { No } \\
\text { cumplen } \\
\text { por } \\
\text { MAYOR } \\
\text { consumo }\end{array}$ & & $\begin{array}{c}\text { Si } \\
\text { cumplen }\end{array}$ & $\begin{array}{c}\text { No } \\
\text { cumplen } \\
\text { por } \\
\text { MENOR } \\
\text { consumo }\end{array}$ & $\begin{array}{c}\text { No } \\
\text { cumplen } \\
\text { por } \\
\text { MAYOR } \\
\text { consumo }\end{array}$ & \\
\hline $\begin{array}{l}3 \text { raciones de } \\
\text { fruta/día }\end{array}$ & $8(47)$ & $9(53)$ & $0(0)$ & & $10(59)$ & $7(41)$ & $0(0)$ & & $+2(+12)$ & $-2(-12)$ & $0(0)$ & \\
\hline $\begin{array}{l}1 \text { ración de } \\
\text { hortalizas } \\
\text { crudas/día }\end{array}$ & $10(59)$ & $6(35)$ & $1(6)$ & & $8(47)$ & $3(18)$ & $6(35)$ & & $-2(-15)$ & $-3(-18)$ & $+5(+29)$ & \\
\hline $\begin{array}{l}1 \text { ración de } \\
\text { hortalizas } \\
\text { cocinadas/día }\end{array}$ & $5(29)$ & $11(65)$ & $1(6)$ & & $4(23)$ & $11(65)$ & $2(12)$ & & $-1(-6)$ & $0(0)$ & $+1(+6)$ & \\
\hline $\begin{array}{l}\text { 2-4 raciones } \\
\text { de pescado/ } \\
\text { semana }\end{array}$ & $12(71)$ & $4(23)$ & $1(6)$ & & $13(76)$ & $2(12)$ & $2(12)$ & & $+1(+6)$ & $-2(-12)$ & $+1(+6)$ & \\
\hline $\begin{array}{l}\text { 2-4 raciones de } \\
\text { carne/semana }\end{array}$ & $8(47)$ & $0(0)$ & $9(53)$ & & $10(59)$ & $0(0)$ & $7(41)$ & & $+2(+12)$ & $0(0)$ & $-2(-12)$ & \\
\hline $\begin{array}{l}\text { 2-4 raciones } \\
\text { de legumbres/ } \\
\text { semana }\end{array}$ & $13(76)$ & $3(18)$ & $1(6)$ & & $8(47)$ & $9(53)$ & $0(0)$ & & $-5(-29)$ & $+6(+35)$ & $-1(-6)$ & \\
\hline $\begin{array}{l}\text { 2-4 raciones } \\
\text { de pasta y/o } \\
\text { arroz/semana }\end{array}$ & $11(65)$ & $5(29)$ & $1(6)$ & & $11(65)$ & $6(35)$ & $0(0)$ & & $0(0)$ & $+1(+6)$ & $-1(-6)$ & \\
\hline $\begin{array}{l}2 \text { raciones de } \\
\text { lácteos/día }\end{array}$ & $7(41)$ & $10(59)$ & $0(0)$ & & $9(53)$ & $8(47)$ & $0(0)$ & & $+2(+12)$ & $-2(-12)$ & $0(0)$ & \\
\hline
\end{tabular}

participantes sea consistente con el patrón español actual ${ }^{11}$ permite considerarlos como válidos.

Los estudios publicados que llevan a cabo una intervención dietético-nutricional en el lugar de trabajo se centran en valorar cambios en la ingesta de frutas y/o hortalizas o en el aporte de grasa de la dieta ${ }^{5,12,13}$. En este sentido, la aparente mejoría encontrada en el presente estudio para estos grupos de alimentos queda corroborada por la evidencia existente y hace pensar que las pequeñas mejoras de los otros grupos también son consistentes. Sólo las legumbres mostraron un empeoramiento, sin embargo, este resultado aparentemente negativo se atribuye a la introducción y explicación del concepto "ración" en los talleres, ya que algunos estudios sugieren que existe una percepción distorsionada de lo que son tamaños de ración normales 0 apropiados ${ }^{14}$. Respecto al cumplimiento con el número y composición de las comidas realizadas, no se conocen otros trabajos en el ámbito laboral que aporten datos con los que comparar. 
Figura 2. Componentes de las comidas de la mañana, desayuno y almuerzo, antes (A) y después (B) del periodo de intervención dietético-nutricional.

A. En el desayuno + almuerzo incluye:

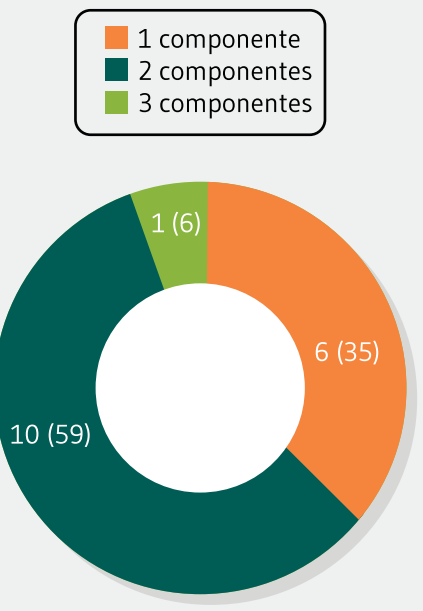

B. En el desayuno incluye:

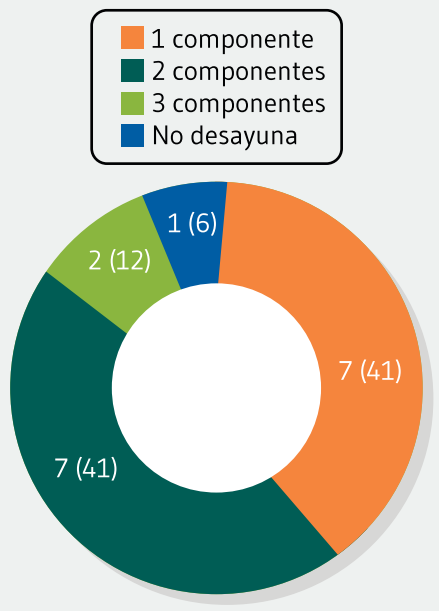

B. En el almuerzo incluye:

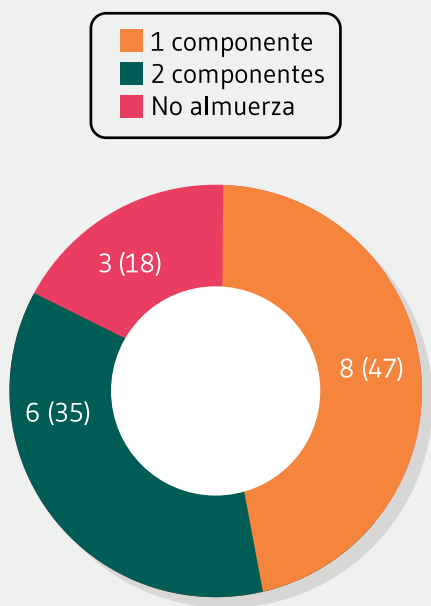

Datos expresados como número (\%). De acuerdo con la SENC ${ }^{10}$, los componentes del desayuno deben ser tres (lácteo, fruta y cereal), mientras que en el almuerzo deben ser dos (combinaciones de los tres componentes del desayuno).

La explicación a este éxito parcial podría venir dada por tres factores. Por un lado, la relativamente corta duración del período de intervención en comparación con otros PPSs ${ }^{5-7,12}$. En segundo lugar, las pocas modificaciones respecto a la disponibilidad de alimentos realizadas por la empresa (en el comedor no se adoptaron completamente los menús recomendados por la dietista-nutricionista y hubo reticencia a cambiar la disponibilidad de alimentos en la zona de almuerzo) ya que cuando se habla sobre la efectividad de las intervenciones dirigidas a modificar comportamientos, además de la educación nutricional, se señala la conveniencia de diseñar estrategias combinadas que aborden también otros aspectos como el entorno ${ }^{5,15}$. Finalmente, el corto período de seguimiento de los participantes para observar los cambios ya que, probablemente, la adaptación de su dieta a los nuevos conocimientos requiere más tiempo.

En cualquier caso, no se puede olvidar la existencia de otros obstáculos de tipo social, cultural y personal que dificultan la adopción de una dieta saludable ${ }^{13}$. Y en este caso, es muy importante considerar la mayoría de hombres entre los participantes, ya que está descrito lo problemático que es para los profesionales de la salud comunicar de forma efectiva con una audiencia masculina así como la alta reticencia que presentan a realizar cambios dietéticos ${ }^{16}$.

Por otro lado, también es necesario resaltar la desigual tasa de asistencia a los planes de formación (el 64\% de los empleados no atendió a los tres planes) así como la mediana participación en la valoración de la eficacia, aunque en el rango reportado en la bibliografía ${ }^{6}$. Tal y como describen Rongen y col. ${ }^{17}$, existen diversos factores que actúan como facilitadores (por ejemplo, apoyo de la empresa, considerarlo agradable/placentero) y como obstáculos (por ejemplo horario de trabajo desfavorable) a la hora de participar en un PPS. Si bien es cierto que la dirección mostró desde el principio una gran predisposición para poder llevar a cabo la intervención dietético-nutricional y que los empleados mostraron satisfacción tras participar en las actividades, no hay que olvidar que el objetivo fundamental de una empresa es la producción, de modo que resulta una prioridad irrefutable. Ante tal situación, aunque se facilitó la documentación escrita para que llegara a todos los empleados, los cambios que se querían lograr se enseñaban con mayor profundidad a través de las charlas por lo que la asistencia a dichos planes de formación también ha podido suponer una limitación para lograr alcanzar los objetivos planificados. 
Es interesante indicar que dos aspectos que se han mostrado como limitaciones del estudio son, al mismo tiempo, dos características que hacen este estudio novedoso. Este PPS dirigido a una empresa pequeña, con una plantilla compuesta básicamente por hombres, aporta luz en un campo de estudio donde predominan las intervenciones con mujeres $^{7}$ y empresas de mayor tamaño ${ }^{8}$.

En conclusión, la intervención dietético-nutricional en esta empresa es compleja y con resultados modestos debido a su propia idiosincrasia y al corto período de tiempo disponible. Aun así, los empleados han adquirido una mayor conciencia de la relación dieta y salud, además de ser una experiencia positiva para la empresa, por lo que la realización de PPSs en este ámbito es prometedora.

\section{• \\ CONFLICTO DE INTERESES}

Los autores expresan que no hay conflictos de intereses al redactar el manuscrito.

\section{BIBLIOGRAFÍA}

1. World Health Organization. Diet, nutrition, and the prevention of chronic diseases: report of a joint WHO/FAO expert consultation. Geneva: World Health Organization; 2003.

2. Ruiz E, del Pozo S, Valero T, Ávila JM, Varela-Moreiras G. Dieta y estado nutricional de la población. En: Libro Blanco de la Nutrición en España. Madrid: Fundación Española de la Nutrición; 2013. p. 31-46.

3. Aranceta Bartrina JA. Nutrición comunitaria. En: Nutrición comunitaria. 3.a ed. Elsevier España; 2013. p. 1-20.

4. World Health Organization. Workers' health: global plan of action [Internet]. WHO; [citado 1 de noviembre de 2015]. Recuperado a partir de: http://www.who.int/occupational_health/ publications/global_plan/en/

5. Ni Mhurchu C, Aston LM, Jebb SA. Effects of worksite health promotion interventions on employee diets: a systematic review. BMC Public Health. 2010; 10: 62.

6. Maes L, Van Cauwenberghe E, Van Lippevelde W, Spittaels $H$,
De Pauw E, Oppert J-M, et al. Effectiveness of workplace interventions in Europe promoting healthy eating: a systematic review. Eur ] Public Health. 2012; 22(5): 677-83.

7. Rongen A, Robroek SJW, van Lenthe FJ, Burdorf A. Workplace health promotion: a meta-analysis of effectiveness. Am J Prev Med. 2013; 44(4): 406-15.

8. McCoy K, Stinson K, Scott K, Tenney L, Newman LS. Health promotion in small business: a systematic review of factors influencing adoption and effectiveness of worksite wellness programs. J Occup Environ Med. 2014; 56(6): 579-87.

9. Sociedad Española de Nutrición Comunitaria, Agencia Española de Seguridad Alimentaria y Nutrición, Dirección General de Educación, Formación Profesional e Innovación Educativa, Centro de Investigación y Documentación Educativa. Cuestionario 3: hábitos alimentarios de la población escolar - Cuestionario para la familia (KidMed) [Internet]. Programa PERSEO - Estrategia Naos; 2007 [citado 1 de noviembre de 2015]. Recuperado a partir de: http://www.perseo.aesan.msssi.gob. es/docs/docs/cuestionarios/cuestionario_3_habitos_alimentarios_poblacion_escolar_familia.pdf

10. Dapcich V, Salvador G, Ribas L, Pérez Rodrigo C, Aranceta Bartrina J, Serra L. Recomendaciones alimentarias: cómo planificar una alimentación saludable. En: Guía de la alimentación saludable. Madrid: Sociedad Española de Nutrición Comunitaria; 2004. p. 8-23.

11. Varela-Moreiras G, Ruiz E, Valero T, Avila JM, del Pozo S. The Spanish diet: an update. Nutr Hosp. 2013; 28 Suppl 5: 13-20.

12. Soler RE, Leeks KD, Razi S, Hopkins DP, Griffith M, Aten A, et al. A systematic review of selected interventions for worksite health promotion. The assessment of health risks with feedback. Am J Prev Med. 2010; 38(2 Suppl): S237-62.

13. Geaney F, Kelly C, Greiner BA, Harrington JM, Perry IJ, Beirne $P$. The effectiveness of workplace dietary modification interventions: a systematic review. Prev Med. 2013; 57(5): 438-47.

14. Macdiarmid Jl, Loe J, Kyle J, McNeill G. «lt was an education in portion size». Experience of eating a healthy diet and barriers to long term dietary change. Appetite. 2013; 71: 411-9.

15. Novoa Pardo AM. Cómo cambiar comportamientos y no morir en el intento: más entornos favorables y menos educación sanitaria. Gac Sanit. 2013; 27(1): 75-6.

16. Newcombe MA, McCarthy MB, Cronin JM, McCarthy SN. «Eat like a man». A social constructionist analysis of the role of food in men's lives. Appetite. 2012; 59(2): 391-8.

17. Rongen A, Robroek SJW, van Ginkel W, Lindeboom D, Altink B, Burdorf $A$. Barriers and facilitators for participation in health promotion programs among employees: a six-month followup study. BMC Public Health. 2014; 14: 573. 